\title{
International study on inter-reader variability for circulating tumor cells in breast cancer
}

\author{
Michail Ignatiadis ${ }^{1,2^{*}}$, Sabine Riethdorf ${ }^{3}$, François-Clement Bidard ${ }^{4}$, Isabelle Vaucher ${ }^{4}$, Mustapha Khazour ${ }^{4}$, \\ Françoise Rothé ${ }^{2}$, Jessica Metallo², Ghizlane Rouas ${ }^{2}$, Rachel E Payne ${ }^{5}$, Raoul Charles Coombes ${ }^{5}$, Ingrid Teufel ${ }^{6}$, \\ Ulrich Andergassen ${ }^{7}$, Stella Apostolaki ${ }^{8}$, Eleni Politaki ${ }^{8}$, Dimitris Mavroudis ${ }^{8}$, Silvia Bessi ${ }^{9}$, Marta Pestrin ${ }^{9}$, \\ Angelo Di Leo ${ }^{9}$, Michael Campion ${ }^{10}$, Monica Reinholz ${ }^{10}$, Edith Perez ${ }^{10}$, Martine Piccart ${ }^{1,2}$, Elin Borgen ${ }^{11}$, \\ Bjorn Naume ${ }^{12}$, Jose Jimenez ${ }^{13}$, Claudia Monica Aura ${ }^{13}$, Laura Zorzino ${ }^{14}$, Maria Cristina Cassatella ${ }^{14}$, \\ Maria Teresa Sandri ${ }^{14}$, Bianca Mostert ${ }^{15}$, Stefan Sleijfer ${ }^{15}$, Jaco Kraan ${ }^{15}$, Wolfgang Janni ${ }^{16}$, Tanja Fehm ${ }^{17}$, \\ Brigitte Rack ${ }^{7}$, Leon Terstappen ${ }^{18}$, Madeline Repollet ${ }^{19}$, Jean-Yves Pierga ${ }^{4}$, Craig Miller ${ }^{19}$, Christos Sotiriou ${ }^{1,2}$, \\ Stefan Michiels ${ }^{20}$ and Klaus Pantel ${ }^{3}$
}

\begin{abstract}
Introduction: Circulating tumor cells (CTCS) have been studied in breast cancer with the CellSearch ${ }^{\circledR}$ system. Given the low CTC counts in non-metastatic breast cancer, it is important to evaluate the inter-reader agreement.

Methods: CellSearch ${ }^{\otimes}$ images ( $N=272$ ) of either $C T C$ s or white blood cells or artifacts from 109 non-metastatic $(M 0)$ and 22 metastatic (M1) breast cancer patients from reported studies were sent to 22 readers from 15 academic laboratories and 8 readers from two Veridex laboratories. Each image was scored as No CTC vs CTC HER2- vs CTC HER2+. The 8 Veridex readers were summarized to a Veridex Consensus (VC) to compare each academic reader using \% agreement and kappa (K) statistics. Agreement was compared according to disease stage and CTC counts using the Wilcoxon signed rank test.
\end{abstract}

Results: For CTC definition (No CTC vs CTC), the median agreement between academic readers and VC was 92\% (range 69 to $97 \%$ ) with a median $\mathrm{k}$ of 0.83 (range 0.37 to 0.93 ). Lower agreement was observed in images from M0 (median 91\%, range 70 to 96\%) compared to M1 (median 98\%, range 64 to 100\%) patients $(P<0.001)$ and from M0 and <3CTCs (median 87\%, range 66 to 95\%) compared to M0 and $\geq 3$ CTCs samples (median 95\%, range 77 to 99\%), $(P<0.001)$ ). For CTC HER2 expression (HER2- vs HER2+), the median agreement was $87 \%$ (range 51 to 95\%) with a median $\mathrm{k}$ of 0.74 (range 0.25 to 0.90 ).

Conclusions: The inter-reader agreement for CTC definition was high. Reduced agreement was observed in M0 patients with low CTC counts. Continuous training and independent image review are required.

\section{Introduction}

Circulating tumor cells (CTCs) can be identified in the peripheral blood of patients with solid tumors even in early disease stages and their detection and characterization have the potential to lead towards personalized treatment strategies in breast and other cancers [1-3].

Several technologies exist for CTC detection, but CellSearch $^{\circ}$ (Veridex, Raritan, NJ, USA) is the only one that

\footnotetext{
*Correspondence: michail.ignatiadis@bordet.be

'Department of Medical Oncology, Institut Jules Bordet, Université Libre de

Bruxelles, Brussels, Belgium

${ }^{2}$ Breast Cancer Translational Research Laboratory, Institut Jules Bordet,

Université Libre de Bruxelles, Brussels, Belgium

Full list of author information is available at the end of the article
}

has received Food and Drug Administration (FDA) clearance to be used as an aid in monitoring patients with metastatic breast, colorectal and prostate cancer. Compared to other CTC assays [4], CellSearch is so far the only semi-automated system and has contributed considerably to the development of standards for CTC enumeration. In breast cancer, a multicenter study has shown that $\geq 5$ CTCs $/ 7.5 \mathrm{ml}$ of blood were detected in $49 \%$ of 177 patients before starting a new treatment for metastatic disease and their detection was independently associated with worse progression-free and overall survival (PFS and OS) [5]. Subsequently, other studies have confirmed these results [6-8]. The value of CTC detection by CellSearch ${ }^{\circ}$ in non-metastatic breast cancer

\section{() Biomed Central}


has recently been emerging. Indeed, detection of $\geq 1 \mathrm{CTC} /$ $7.5 \mathrm{ml}$ was reported in $23 \%$ of 115 patients with large operable and locally advanced breast cancer before neoadjuvant chemotherapy and was independently associated with worse outcome [9]. In another study, detection of $\geq 1$ CTC/ $7.5 \mathrm{ml}$ by CellSearch ${ }^{\circ}$ at the time of surgery and before the administration of adjuvant chemotherapy in $24 \%$ of 302 patients was associated with decreased PFS and OS [10]. In the SUCCESS study, $\geq 1 \mathrm{CTC} / 23 \mathrm{ml}$ were detected by CellSearch $^{\circ}$ in $21.5 \%$ of 2,026 patients with early breast cancer before adjuvant chemotherapy and the detection was independently associated with poor outcome [11]. In another study $\geq 1 \mathrm{CTC} / 30 \mathrm{ml}$ of blood were detected in 19\% of 404 patients with stage-I to -III breast cancer before surgery and their detection was independently associated with shorter distant disease-free survival [12]. Beyond enumeration, CTC characterization holds the promise to serve as a liquid biopsy to tailor treatment decisions [1,2]. As an example, as HER2 protein overexpression or gene amplification in the primary tumor is currently required for administering antiHER2 treatment in breast cancer [13], HER2 status on CTCs has also been investigated in breast cancer using CellSearch $^{\circ}$ and other technologies [14-18].

More than 400 studies have included CTCs as a biomarker [19]. These include interventional studies assessing the value of CTCs as a treatment decision tool in the metastatic and non-metastatic setting [20,21]. Two studies have validated the analytical performance of CellSearch ${ }^{\circ}$ for CTC detection in metastatic breast cancer [22,23]. In the metastatic setting, the main contributor to interlaboratory variation was variability among the readers in image interpretation [23,24]. In non-metastatic breast cancer (BC) more than half of women with detectable CTCs have only $1 \mathrm{CTC} /$ blood volume processed [9-12]. Therefore, in this setting, image interpretation is crucial, especially if CTC detection is to be used as a tool to decide on secondary adjuvant treatment strategies in the context of a clinical trial. Moreover, no study has addressed potential problems in image interpretation when HER2 expression on CTCs is investigated using CellSearch ${ }^{\circ}$, which is an important prerequisite for interventional studies using CTCs in trials testing HER2-directed therapies.

Therefore, we performed an international study to evaluate the inter-reader agreement in the classification of CellSearch ${ }^{\circ}$ images as CTC and interpretation of HER2staining on these images. Moreover, we came up with consensus guidelines for image interpretation in the nonmetastatic breast cancer setting that were subsequently endorsed by CTC experts participating in this study.

\section{Methods}

\section{Patient and samples}

CellSearch images from blood samples used for the detection of CTCs and characterization of CTC HER2 protein expression by CellSearch ${ }^{\circ}$ from breast cancer patients were retrieved for this study. The blood samples were processed at one of the three different laboratories; the Institut Jules Bordet, Belgium (IJB), the Institut Curie, France (IC), and the University of Hamburg, Germany $(\mathrm{UH})$, respectively. The IJB dataset included CellSearch ${ }^{\circ}$ images from women with non-metastatic (M0) and metastatic (M1) breast cancer treated according to the standard of care in Belgium [17]. The IC dataset consisted of CellSearch ${ }^{\ominus}$ images from women with inflammatory M0 breast cancer participating in the BEVERLY-I and -II phase-2 trials $[25,26]$. The UH dataset included CellSearch ${ }^{\circ}$ images from women receiving neoadjuvant chemotherapy in the GeparQuattro and GeparQuinto studies (M0 cohort) $[16,27]$ and women from the Detect Study (M1 cohort) [18]. From each patient sample, a subset of CellSearch ${ }^{\circ}$ images (images of either CTCs or white blood cells or artifacts) and not the full set of CellSearch images were provided by investigators from the three independent, academic labs to the principal investigator at IJB who was responsible for the central coordination of the study. Due to technology limitations, at the time the study was initiated, it was not possible to send the full set of CellSearch images from each patient sample. All CellSearch ${ }^{\bullet}$ images used in the present study are from previously reported studies of CTC detection in breast cancer [16-18,25-27]. All women participating in these studies provided informed consent and each study was approved by the respective institutions' ethical committees. The present study was approved by the IJB ethical committee (CE 2035). Informed consent for the present study was waived by the IJB ethical committee.

\section{Readers}

The IJB lab sent the blinded CellSearch ${ }^{\circ}$ images by email to twenty-two readers from one US and fourteen European academic laboratories as well as to eight readers from two Veridex laboratories. Each CellSearch image was evaluated independently of the other images. All readers had already received the appropriate training provided by Veridex for the CellSearch ${ }^{\circ}$ system, which includes training in image interpretation.

\section{Definition of CTC and HER2-positive CTC}

In CellSearch ${ }^{\circ}$ a CTC is defined as an epithelial cell adhesion molecule (EpCAM)-positive cell (round to oval and sometimes polygonal or elongated in shape and at least $4 \mu \mathrm{m}$ in size) that has positive immunofluoresence staining for a Cytokeratin (CK) (clone C11 and A53-B/A2) epithelial marker and positive staining for the nuclear dye 4',6-diamidino-2-phenylindole (DAPI) (the nuclear area should be smaller than the cytoplasmic area and at least $50 \%$ of the nucleus should be co-located with the cytoplasm). The CTC must also be negative for the leukocyte 
marker CD45. For this study, the readers were asked to score CTCs with HER2 immunofluoresence staining intensity of 2+, 3+ as HER2-positive and were provided with reference CellSearch images from breast cancer cell lines (MCF7, BT20, T47D, MDA-MB-453, SKBR3, BT474) with different levels of HER2 expression according to Riethdorf et al. [16]. The readers scored each image as follows: No CTC, CTC/HER2-negative (CTC HER2-), or CTC/HER2positive (CTC HER2+).

\section{Statistics}

Results from the eight Veridex readers were summarized to a Veridex consensus (VC) in order to compare each academic reader using percentage (\%) agreement and kappa (к) statistics. We followed the Koch and Landis classification to interpret obtained $\mathrm{K}$ values: concordance was considered bad if $\mathrm{K}$ was less than 0.20 , poor if $\mathrm{K}$ was 0.20 to 0.40 , moderate if $\kappa$ was 0.40 to 0.60 , good if $\kappa$ was 0.60 to 0.80 , and almost perfect if $\mathrm{K}$ was above 0.81 [28]. $\mathrm{VC}$ was reached when there was $>60 \%$ agreement between Veridex readers as to whether or not an image was a CTC. When there was $<60 \%$ agreement by the Veridex readers for an image, this image was not included in any of the subsequent comparisons. If the $\mathrm{VC}$ was that the image was a CTC, then there had to be $>60 \%$ agreement on the CTC being either HER2- or HER2+. If there was $<60 \%$ agreement about the HER2 positivity or negativity among the Veridex readers for a CTC, this image was not included in any of the subsequent comparisons.

For each of the 22 academic readers, percentage (\%) agreement with $\mathrm{VC}$ was calculated for the CTC detection question (no CTC versus CTC) and for the question of HER2 expression on CTCs (CTC HER2- versus CTC HER2+). In order to understand problems related to CellSearch ${ }^{\circ}$ image interpretation, we compared the locations in the distributions of the agreement (\%) between VC and the academic readers according to the dataset (IJB versus $\mathrm{IC}$ versus $\mathrm{UH}$ ), disease stage (M0 versus $\mathrm{M} 1$ ), number of detected CTCs in the entire sample $(\geq 5$ CTCs versus $<5$ CTCs) and administration of systemic treatment before blood sampling (yes versus no) using non-parametric tests (Wilcoxon signed rank and Freedman test). Moreover, within M0 patient samples, we interrogated whether there was different agreement according to the number of CTCs detected in the entire sample ( $\geq 3$ CTCs versus $<3$ CTCs) using the Wilcoxon signed rank test. Finally, we studied CellSearch ${ }^{\circ}$ images with $<70 \%$ agreement between VC and academic readers in order to understand reasons for discordance between readers.

\section{Results}

CellSearch ${ }^{\circledast}$ images and patient characteristics

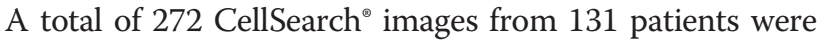
analyzed. Table 1 provides information on relevant study
Table 1 CellSearch ${ }^{\circledR}$ images and patient characteristics

\begin{tabular}{cc}
\hline $\begin{array}{c}\text { Patients, } \\
\text { number }(\%)\end{array}$ & $\begin{array}{c}\text { CellSearch }{ }^{\circledast} \text { images, } \\
\text { number }(\%)\end{array}$ \\
$\mathrm{n}=131(100 \%)$ & $\mathrm{n}=\mathbf{2 7 2}(100 \%)$ \\
\hline
\end{tabular}

\begin{tabular}{lcc}
\hline Dataset & & \\
Institute Jules Bordet & $34(26)$ & $60(22)$ \\
Institute Curie & $60(46)$ & $114(42)$ \\
University of Hamburg & $37(28)$ & $98(36)$ \\
Age, years & & \\
$\leq 50$ & $58(44)$ & $108(40)$ \\
$>50$ & $66(51)$ & $147(54)$ \\
Unknown & $7(5)$ & $17(6)$ \\
ER & & $106(39)$ \\
Negative & $54(41)$ & $139(51)$ \\
Positive & $68(52)$ & $27(10)$ \\
Unknown & $9(7)$ &
\end{tabular}

HER2

$\begin{array}{lcc}\text { Negative } & 103(79) & 193(71) \\ \text { Positive } & 15(11) & 44(16) \\ \text { Unknown } & 13(10) & 35(13)\end{array}$

Histology grade

$\begin{array}{lcc}1 & 5(4) & 6(2) \\ 2 & 46(35) & 85(31) \\ 3 & 69(53) & 147(54) \\ \text { Unknown } & 11(8) & 34(13)\end{array}$

Histology

$\begin{array}{lll}\text { Ductal } & 99(76) & 202(74)\end{array}$

Lobular $10(8) \quad 12(4)$

$\begin{array}{ll}\text { Other } & 7(5)\end{array}$

Unknown $15(11) \quad 40(15)$

Stage

M0 $109(83) \quad 215(79)$

$\begin{array}{lll}\text { M1 } & 22(17) & 57(21)\end{array}$

CTC count ${ }^{1}$

$<5$ CTCs $\quad 90(69) \quad 150(55)$

$\geq 5$ CTCs $\quad 40(30) \quad 120(44)$

Unknown 2 (1) 2 (1)

Systemic therapy before blood sample collection ${ }^{2}$

$\begin{array}{lll}\text { No } & 87(65) & 168(62) \\ \text { Yes } & 31(24) & 62(23) \\ \text { Unknown } & 15(11) & 42(15)\end{array}$

${ }^{1}$ One patient with two samples ( $<5$ CTCs and $\geq 5$ CTCs) was included; ${ }^{2}$ two patients with samples before and after treatment were included. ER, estrogen receptor; CTC, circulating tumor cell; M0, non-metastatic; M1, metastatic.

demographics. The majority of CellSearch ${ }^{\circ}$ images analyzed were from patients with M0 breast cancer, with $<5$ CTCs in their blood sample and from patients for whom 
blood sampling for CTC detection was performed before the administration of systemic treatment (Table 1).

\section{Veridex consensus (VC)}

For each image, the results from the eight Veridex readers were summarized to a $\mathrm{VC}$, as explained in the Methods section. For CTC detection, VC was reached for 267 (98\%) of 272 images with an average percent agreement for CTC versus no CTC between the eight Veridex readers of $97 \%$ (median 100\%, range 63\% to 100\%). Of the 267 images, 157 (59\%) were considered CTCs. For interpretation of HER2 staining on the CTC, VC was obtained for 155 (99\%) out of 157 CTCs with an average percent agreement for HER2- versus HER2+ between the eight Veridex readers of $98 \%$ (median 100\%, range $63 \%$ to $100 \%$ ): 71 (46\%) of 155 CTCs were considered as HER2 +.

\section{Agreement between academic readers and Veridex consensus for CTC definition}

For the question of whether an image was a CTC (no CTC versus CTC), the median agreement between the academic readers and VC was $92 \%$ (range 69\% to 97\%), with a median $\mathrm{k}$ statistic of 0.83 (range 0.37 to 0.93 ) (Table 2). We then asked whether agreement between the academic readers and $\mathrm{VC}$ was different according to the dataset (IJB versus IC versus UH), disease stage (M0 versus M1), CTC counts ( $<5$ versus $\geq 5$ ) and administration of systemic treatment before blood sampling (yes versus no). We observed significantly lower agreement between academic readers and VC for CellSearch ${ }^{\circ}$ images from patients of the IJB dataset compared to the other datasets, from patients with M0 compared to M1 disease, $<5$ CTCs compared to $\geq 5$ CTCs, and blood samples drawn after the administration of systemic treatment as compared to samples drawn before systemic treatment (Table 3, Additional file 1: Table S1 and Additional file 2: Table S2, online). CellSearch ${ }^{\circ}$ images from samples with $<5$ CTCs were present more frequently in M0 (146 of 213 images, 68\%) compared to M1 patients (4 of 57 images, 7\%) (Pearson chi-square test, $P<0.001)$ and in the IJB dataset, (52 of 60 images, $87 \%$ ) compared to the UH (22 of 96 images, $22 \%$ ) and the IC dataset (76 of 114 images, 67\%) $(P<0.001)$. When only images from Mo patients samples were

Table 2 Agreement (\%) between academic readers and Veridex consensus (VC)

\begin{tabular}{|c|c|c|c|c|c|c|c|c|}
\hline \multirow[b]{2}{*}{ RDs } & \multicolumn{2}{|c|}{ No CTC versus CTC } & \multirow[b]{2}{*}{ Kappa statistic } & \multirow[b]{2}{*}{ Kappa agreement } & \multicolumn{3}{|c|}{ CTC HER2- versus CTC HER2+ } & \multirow[b]{2}{*}{ Kappa agreemen } \\
\hline & $N$ & Agreement (\%) & & & $\mathbf{N}$ & Agreement (\%) & Kappa statistic & \\
\hline A & 267 & $91.8 \%$ & 0.83 & Almost perfect & 155 & $81.3 \%$ & 0.63 & Substantial \\
\hline B & 267 & $91.4 \%$ & 0.82 & Almost perfect & 155 & $81.3 \%$ & 0.63 & Substantial \\
\hline C & 267 & $92.5 \%$ & 0.84 & Almost perfect & 155 & $90.3 \%$ & 0.81 & Almost perfect \\
\hline $\mathrm{D}$ & 267 & $91.8 \%$ & 0.83 & Almost perfect & 155 & $71.6 \%$ & 0.47 & Moderate \\
\hline $\mathrm{E}$ & 266 & $95.1 \%$ & 0.90 & Almost perfect & 154 & $93.5 \%$ & 0.87 & Almost perfect \\
\hline $\mathrm{F}$ & 267 & $92.1 \%$ & 0.84 & Almost perfect & 155 & $87.1 \%$ & 0.75 & Substantial \\
\hline G & 267 & $91.4 \%$ & 0.82 & Almost perfect & 155 & $91.0 \%$ & 0.82 & Almost perfect \\
\hline $\mathrm{H}$ & 267 & $91.4 \%$ & 0.82 & Almost perfect & 155 & $88.4 \%$ & 0.78 & Substantial \\
\hline I & 267 & $92.1 \%$ & 0.84 & Almost perfect & 155 & $91.6 \%$ & 0.84 & Almost perfect \\
\hline J & 267 & $83.9 \%$ & 0.65 & Substantial & 155 & $76.8 \%$ & 0.55 & Moderate \\
\hline K & 220 & $87.3 \%$ & 0.74 & Substantial & 117 & $79.5 \%$ & 0.59 & Moderate \\
\hline L & 266 & $92.9 \%$ & 0.85 & Almost perfect & 154 & $94.8 \%$ & 0.90 & Almost perfect \\
\hline M & 220 & $87.3 \%$ & 0.74 & Substantial & 117 & $90.6 \%$ & 0.82 & Almost perfect \\
\hline $\mathrm{N}$ & 267 & $95.9 \%$ & 0.92 & Almost perfect & 155 & $92.3 \%$ & 0.85 & Almost perfect \\
\hline $\mathrm{O}$ & 267 & $96.6 \%$ & 0.93 & Almost perfect & 155 & $92.9 \%$ & 0.86 & Almost perfect \\
\hline$P$ & 267 & $94.4 \%$ & 0.88 & Almost perfect & 155 & $85.2 \%$ & 0.72 & Substantial \\
\hline Q & 218 & $91.7 \%$ & 0.83 & Almost perfect & 113 & $84.1 \%$ & 0.69 & Substantial \\
\hline R & 218 & $91.7 \%$ & 0.83 & Almost perfect & 113 & $85.8 \%$ & 0.73 & Substantial \\
\hline S & 220 & $92.7 \%$ & 0.85 & Almost perfect & 117 & $86.3 \%$ & 0.73 & Substantial \\
\hline $\mathrm{T}$ & 266 & $69.2 \%$ & 0.37 & Fair & 154 & $51.3 \%$ & 0.25 & Fair \\
\hline U & 267 & $91.4 \%$ & 0.82 & Almost perfect & 155 & $91.0 \%$ & 0.82 & Almost perfect \\
\hline V & 220 & $91.8 \%$ & 0.84 & Almost perfect & 117 & $82.1 \%$ & 0.66 & Substantial \\
\hline
\end{tabular}

CTC, circulating tumor cell; RDs, readers; N, number of images. Kappa Agreement, below 0.00 Poor; 0.00 to 0.20 Slight; 0.21 to 0.40 Fair; 0.41 to 0.60 Moderate; 0.61 to 0.80 Substantial; 0.81 to 1.00 Almost Perfect. 
analyzed, lower agreement was observed for images from samples with $<3$ CTCs (median $87 \%$, range $66 \%$ to $95 \%$ ) compared to those from samples where $\geq 3$ CTCs were detected (median $95 \%$, range $77 \%$ to $99 \%),(P<0.001)$.

\section{Agreement between academic readers and Veridex consensus (VC) for HER2 expression on CTCs}

When only the images that were CTCs according to VC were analyzed for HER2 protein expression (CTC HER2versus CTC HER2+), the median agreement between the academic readers and VC was $87 \%$ (range $51 \%$ to $95 \%$ ), with a median $\mathrm{K}$ statistic of 0.74 (range 0.25 to 0.90 ) (Table 2).

\section{Analysis of CellSearch ${ }^{\circledast}$ images with $<70 \%$ agreement} between academic readers and Veridex consensus (VC) There were many images with excellent agreement between academic readers and $\mathrm{VC}$, such as images of intact CTCs (Figure 1A). In order to understand the reasons for discordance in image interpretation, we focused on images with $<70 \%$ agreement between the academic readers and VC. We identified 25 images with $<70 \%$ agreement for the CTC question (CTC versus no CTC) and 14 images with $<70 \%$ agreement for the HER2 expression question (HER2- versus HER2+ CTCs). Disagreement was observed for images of CTCs with CK staining that surrounded the nucleus but was either incomplete or granular (Figure 1B). A total of 16 of the 25 images

\begin{tabular}{|c|c|c|c|}
\hline & \multicolumn{2}{|c|}{ Agreement, \% } & \multirow[b]{2}{*}{$P$-value } \\
\hline & Median & Range & \\
\hline$\overline{\text { All }}$ & 92 & 69 to 97 & \\
\hline \multicolumn{4}{|l|}{ Dataset } \\
\hline Institute Jules Bordet & 81 & 61 to 97 & $<0.001$ \\
\hline Institute Curie & 97 & 73 to 99 & \\
\hline University of Hamburg & 94 & 69 to 98 & \\
\hline \multicolumn{4}{|l|}{ Stage } \\
\hline MO & 91 & 70 to 96 & $<0.001$ \\
\hline M1 & 98 & 64 to 100 & \\
\hline \multicolumn{4}{|l|}{ CTC count } \\
\hline$<5 \mathrm{CTCS}$ & 88 & 66 to 95 & $<0.001$ \\
\hline$\geq 5 \mathrm{CTCs}$ & 97 & 74 to 100 & \\
\hline \multicolumn{4}{|l|}{$\begin{array}{l}\text { Systemic therapy before } \\
\text { blood sample collection }\end{array}$} \\
\hline No & 92 & 74 to 96 & $<0.001$ \\
\hline Yes & 89 & 55 to 97 & \\
\hline
\end{tabular}

CTC, circulating tumor cell; M0, Non-metastatic, M1, metastatic. with $<70 \%$ agreement for the CTC question were considered by some academic readers as CTCs, whereas the $\mathrm{VC}$ was that these images were not CTCs. These included images displaying the same morphology in all channels (Figure 1C,D) or a nucleus larger than the cytoplasm or $<50 \%$ inside the cytoplasm and with cytokeratin (CK) staining not surrounding the nucleus (Figure 1E,F). Images with $<70 \%$ agreement between academic readers and $\mathrm{VC}$ for the HER2 expression question were images from CTCs with intermediate HER2 staining (neither too strong nor absent).

\section{Discussion}

To our knowledge, this is the first study to evaluate the inter-reader agreement for CTC detection in both M1 and $\mathrm{M} 0$ breast cancer in a large international multicenter study comprising 15 centers from Europe and the United States. Our results show that the inter-reader agreement is high for CTC definition and for characterizing HER2 expression on CTCs.

Lower inter-reader agreement for CTC definition was observed for CellSearch images of samples from patients with M0 disease, with low CTC counts, samples from the IJB dataset, and samples drawn after the administration of systemic treatment. CellSearch ${ }^{\bullet}$ images of samples with low CTC counts were more frequently observed in the IJB compared to the other datasets and in M0 compared to M1 disease and this can partly explain the lower inter-reader agreement in these groups. In a recently reported study of CTC detection by CellSearch $^{\circ}$ at the time of surgery in patients with early breast cancer, increasing CTC counts were associated with increasing hazard ratio (HR) for disease progression. The presence of $\geq 1$ CTC, $\geq 2$ CTCs and $\geq 3$ CTCs was associated with a HR of 4.6, 5.5 and 6.7, respectively [10]. Although, this increase in HRs can be attributed to the increase in the number of CTCs, the data from the present analysis imply that this might be also due to the lower false CTC-positive cases in women with increasing CTC counts. Finally, a possible explanation for the lower inter-reader agreement in samples drawn after the administration of systemic treatment is the presence in these samples of granular CTCs that have been demonstrated to be mainly apoptotic, and to be a source of inter-reader disagreement in a previous study [23].

There are some limitations in the way the present validation study was conducted. Although CellSearch ${ }^{\circ}$ image selection was performed by three independent, academic laboratories, a selection bias towards either CellSearch ${ }^{\circ}$ images that are easy (typical CTC images) or difficult to interpret (not typical CTC images) cannot be excluded. Moreover, the readers were provided with a subset of CellSearch $^{\circ}$ images (images of either CTCs or white blood cells or artifacts) and not the full set of CellSearch images 


\begin{tabular}{|c|c|c|c|c|c|c|}
\hline & & Composite & CK & DAPI & CD45 & HER2 \\
\hline Intact CTC & A. & & & & & \\
\hline Granular CTC & B. & $8 z$ & $8 ;$ & & & \\
\hline No CTC & c. & $\mathbf{s}$ & 8 & $\mathbf{8}$ & & 8 \\
\hline No CTC & D. & 2 & 8 & & & 8 \\
\hline No CTC & E. & & 6 & & & \\
\hline No CTC & F. & 80 & b) & & & \\
\hline
\end{tabular}

from each patient sample. Therefore, a bias in image selection cannot be excluded. Moreover, the readers were scoring images but were not able to use the Cell Select tool of the CellSearch system, which allows relocation of a cellular image in the cartridge and study of its characteristics in more detail. As the technology evolves, one can imagine that the exchange of CellSearch ${ }^{\circ}$ images for central image review will be more easily performed.

The above limitations may partly explain some of the discrepancies observed between readers. Although overall inter-reader agreement was high, agreement was lower in images from M0 patients with $<3$ CTCs. In order to improve the performance of academic readers, especially in the setting of non-metastatic breast cancer with low CTC counts, we propose the following: 1) images displaying identical morphology in all channels (CK, DAPI and HER2 if performed) or images with a nucleus larger than the cytoplasm or $<50 \%$ inside the cytoplasm should not be considered as CTCs; 2) the authors reporting studies on
CTCs using CellSearch ${ }^{\bullet}$ should clearly indicate whether their CTC definition includes only intact CTCs (complete CK staining surrounding the nucleus) or whether it also includes granular CTCs (CK staining that surrounds the nucleus but is either granular or incomplete); 3 ) in cases of blood samples from M0 patients with low CTC counts ( $<3$ CTCs) evaluation of images by at least two independent readers - ideally from different labs - should be performed. Such an independent image review is applied in the ongoing European Organization for Research and Treatment of Cancer (EORTC) Treat CTC trial (ClinicalTrials.gov Identifier: NCT01548677). This trial is testing the value of CTC detection to decide for secondary adjuvant treatment in $\mathrm{BC}$.

We propose that investigators conducting studies on CTCs in non-metastatic breast cancer follow these guidelines for CellSearch ${ }^{\bullet}$ image interpretation. The present study might also be a model for similar experiments regarding other image-based CTC technologies. This could 
facilitate consistent result interpretation across studies on CTC detection and characterization, and accelerate the clinical development of CTC-related biomarkers.

\section{Conclusions}

This is the first study to evaluate the inter-reader agreement for CTC detection in both metastatic and nonmetastatic $\mathrm{BC}$ in a large international multicenter study comprising 15 centers from Europe and the United States. We demonstrated that inter-reader agreement using CellSearch $^{\circ}$ was high overall but was reduced in nonmetastatic breast cancer patients with low CTC counts. This study resulted in consensus guidelines for image interpretation for CTC detection in non-metastatic breast cancer. Continuous training, independent image review and adherence to these consensus guidelines should be considered in studies evaluating the clinical utility of CTC-related biomarkers in non-metastatic breast cancer.

\section{Additional files}

Additional file 1: Table S1. Agreement (\%) between academic readers and Veridex consensus (VC) according to dataset and disease stage. Description of data: we observed significantly lower agreement between academic readers and VC for CellSearch ${ }^{\oplus}$ images from patients of the Institut Jules Bordet (IJB) dataset compared to the other datasets and from patients with non-metastatic (M0) compared to metastatic (M1) disease.

Additional file 2: Table S2. Agreement (\%) between academic readers and Veridex consensus (VC) according to circulating tumor cell (CTC) count and administration of systemic therapy before blood sample collection. Description of data: we observed significantly lower agreement between academic readers and VC for CellSearch ${ }^{\circledast}$ images from patients with $<5$ CTCs compared to $\geq 5$ CTCs and blood samples drawn after the administration of systemic treatment as compared to samples drawn before systemic treatment.

\section{Abbreviations}

BC: breast cancer; CK: cytokeratin; CTCs: circulating tumor cells; DAPI: 4',6-diamidino-2-phenylindole; EORTC: European Organization for Research and Treatment of Cancer; EpCAM: epithelial cell adhesion molecule; FDA: Food and Drug Administration; HR: hazard ratio; IC: Institut Curie; IJB: Institut Jules Bordet; MO: non-metastatic; M1: metastatic; OS: overall survival; PFS: progression-free survival; UH: University of Hamburg; VC: Veridex consensus.

\section{Competing interests}

Michail Ignatiadis, Francois-Clement Bidard, Stefan Sleijfer, Wolfgang Janni, Brigitte Rack, Leon Terstappen, Jean-Yves Pierga and Klaus Pantel have received research support from CellSearch ${ }^{\oplus}$, Veridex. Michail Ignatiadis, Francois-Clement Bidard, Stefan Sleijfer, Leon Terstappen, Jean-Yves Pierga and Klaus Pantel have received consultancy fees from CellSearch ${ }^{\oplus}$, Veridex. All other authors have no conflict of interest to declare.

\section{Authors' contributions}

MI, CM, SM, KP were involved in study conception and design. All authors were involved in acquisition, analysis, or interpretation of data for this study. $\mathrm{Ml}$ wrote the manuscript. All authors provided input by revising the manuscript critically for important intellectual content. All authors read and approved the final version of the manuscript.

\section{Acknowledgements}

Michail Ignatiadis has received grants from Breast Cancer Research Foundation (BCRF), Fonds de la recherche scientifique FNRS, MEDIC foundation and Les Amis de I' Institut Bordet. No further funding was provided for this study.

\section{Author details}

${ }^{1}$ Department of Medical Oncology, Institut Jules Bordet, Université Libre de Bruxelles, Brussels, Belgium. ${ }^{2}$ Breast Cancer Translational Research Laboratory, Institut Jules Bordet, Université Libre de Bruxelles, Brussels, Belgium. ${ }^{3}$ Institute of Tumor Biology, University Medical Center Hamburg-Eppendorf, Hamburg, Germany. ${ }^{4}$ Department of Medical Oncology, Institut Curie \& Université Paris Descartes, Paris, France. ${ }^{5}$ Division of Cancer, Imperial College, Hammersmith Hospital, London, UK. ${ }^{6}$ Department of Gynecology, University of Tubingen, Tubingen, Germany. ${ }^{7}$ Department of Gynecology, Ludwig-Maximilians University of Munich, Munich, Germany. ${ }^{8}$ Department of Medical Oncology, University of Crete, Heraklion, Greece. Pranslational Research Unit, Hospital of Prato, Prato, Italy. ${ }^{10}$ Department of Laboratory Medicine and Pathology, Mayo Clinic, Rochester, USA. ${ }^{11}$ Department of Pathology, The Radium Hospital, Oslo, Norway. ${ }^{12}$ Department of Oncology, Oslo University Hospital and K.G. Jebsen Centre for Breast Cancer Research, Institute for Clinical Medicine, University of Oslo, Oslo, Norway. ${ }^{13}$ Molecular Pathology Lab, Vall d'Hebron Institut de Recerca (VHIR), Barcelona, Spain. ${ }^{14}$ Division of Laboratory Medicine, European Institute of Oncology, Milan, Italy. ${ }^{15}$ Department of Medical Oncology, Erasmus Medical Center, Rotterdam, The Netherlands. ${ }^{16}$ Department of Gynecology, University of Ulm, Ulm, Germany.

${ }^{17}$ Department of Gynecology, University of Düsseldorf, Düsseldorf, Germany.

${ }^{18}$ Department of Medical Cell BioPhysics, Enschede, The Netherlands.

${ }^{19}$ Veridex LCC, Philadelphia, USA. ${ }^{20}$ Department of Biostatistics and Epidemiology, Institut Goustave Roussy, Paris, France.

Received: 4 October 2013 Accepted: 3 April 2014

Published: 23 April 2014

\section{References}

1. Pantel K, Brakenhoff $\mathrm{RH}$, Brandt B: Detection, clinical relevance and specific biological properties of disseminating tumour cells. Nat Rev Cancer 2008, 8:329-340.

2. Ignatiadis M, Reinholz MM: Minimal residual disease and circulating tumor cells in breast cancer. Breast Cancer Res 2011, 13:222.

3. Zhang L, Riethdorf S, Wu G, Wang T, Yang K, Peng G, Liu J, Pantel K: Meta-analysis of the prognostic value of circulating tumor cells in breast cancer. Clin Cancer Res 2012, 18:5701-5710.

4. Alix-Panabieres C, Schwarzenbach $H$, Pantel K: Circulating tumor cells and circulating tumor DNA. Annu Rev Med 2012, 63:199-215.

5. Cristofanilli M, Budd GT, Ellis MJ, Stopeck A, Matera J, Miller MC, Reuben JM, Doyle GV, Allard WJ, Terstappen LW, Hayes DF: Circulating tumor cells, disease progression, and survival in metastatic breast cancer. N Engl Med 2004, 351:781-791.

6. Nolé F, Munzone E, Zorzino L, Minchella I, Salvatici M, Botteri E, Medici M, Verri E, Adamoli L, Rotmensz N, Goldhirsch A, Sandri MT: Variation of circulating tumor cell levels during treatment of metastatic breast cancer: prognostic and therapeutic implications. Ann Oncol 2008, 19:891-897.

7. Liu MC, Mego M, Nakamura S, Nole F, Pierga J, Toi M, Munzone E, Yagata H, Sandri MT, Bidard F, Wang H, Hayes DF, Cristofanilli M: Clinical validity of circulating tumor cell (CTC) enumeration in 841 subjects with metastatic breast cancer (MBC). J Clin Oncol 2011, 29:10592.

8. Pierga JY, Hajage D, Bachelot T, Delaloge S, Brain E, Campone M, Diéras V, Rolland E, Mignot L, Mathiot C, Bidard FC: High independent prognostic and predictive value of circulating tumor cells compared with serum tumor markers in a large prospective trial in first-line chemotherapy for metastatic breast cancer patients. Ann Oncol 2012, 23:618-624.

9. Bidard FC, Mathiot C, Delaloge S, Brain E, Giachetti S, de Cremoux P, Marty $M$, Pierga JY: Single circulating tumor cell detection and overall survival in nonmetastatic breast cancer. Ann Oncol 2010, 21:729-733.

10. Lucci A, Hall CS, Lodhi AK, Bhattacharyya A, Anderson AE, Xiao L, Bedrosian I, Kuerer HM, Krishnamurthy S: Circulating tumour cells in non-metastatic breast cancer: a prospective study. Lancet Oncol 2012, 13:688-695.

11. Rack B, Andergassen U, Janni W, Neugebauer J: CTCs in primary breast cancer (I). Recent Results Cancer Res 2012, 195:179-185. 
12. Franken B, de Groot MR, Mastboom WJ, Vermes I, van der Palen J, Tibbe AG, Terstappen LW: Circulating tumor cells, disease recurrence and survival in newly diagnosed breast cancer. Breast Cancer Res 2012, 14:R133.

13. Wolff AC, Hammond ME, Schwartz JN, Hagerty KL, Allred DC, Cote RJ, Dowsett M, Fitzgibbons PL, Hanna WM, Langer A, McShane LM, Paik S, Pegram MD, Perez EA, Press MF, Rhodes A, Sturgeon C, Taube SE, Tubbs R, Vance $\mathrm{GH}$, van de Vijver M, Wheeler TM, Hayes DF: American Society of Clinical Oncology; College of American Pathologists. American Society of Clinical Oncology/College of American Pathologists guideline recommendations for human epidermal growth factor receptor 2 testing in breast cancer. J Clin Oncol 2007, 25:118-145.

14. Hayes DF, Walker TM, Singh B, Vitetta ES, Uhr JW, Gross S, Rao C, Doyle GV, Terstappen LW: Monitoring expression of HER-2 on circulating epithelial cells in patients with advanced breast cancer. Int J Oncol 2002, 21:1111-1117.

15. Meng S, Tripathy D, Shete S, Ashfaq R, Haley B, Perkins S, Beitsch P, Khan A, Euhus D, Osborne C, Frenkel E, Hoover S, Leitch M, Clifford E, Vitetta E, Morrison L, Herlyn D, Terstappen LW, Fleming T, Fehm T, Tucker T, Lane N, Wang J, Uhr J: HER-2 gene amplification can be acquired as breast cancer progresses. Proc Natl Acad Sci USA 2004, 101:9393-9398.

16. Riethdorf S, Müller V, Zhang L, Rau T, Loibl S, Komor M, Roller M, Huober J, Fehm T, Schrader I, Hilfrich J, Holms F, Tesch H, Eidtmann H, Untch M, von Minckwitz G, Pantel K: Detection and HER2 expression of circulating tumor cells: prospective monitoring in breast cancer patients treated in the neoadjuvant GeparQuattro trial. Clin Cancer Res 2010, 16:2634-2645.

17. Ignatiadis M, Rothé F, Chaboteaux C, Durbecq V, Rouas G, Criscitiello C Metallo J, Kheddoumi N, Singhal SK, Michiels S, Veys I, Rossari J, Larsimont D, Carly B, Pestrin M, Bessi S, Buxant F, Liebens F, Piccart M, Sotiriou C: HER2-positive circulating tumor cells in breast cancer. PLOS One 2011, 6:e15624

18. Fehm T, Müller V, Aktas B, Janni W, Schneeweiss A, Stickeler E, Lattrich C, Löhberg CR, Solomayer E, Rack B, Riethdorf S, Klein C, Schindlbeck C Brocker K, Kasimir-Bauer S, Wallwiener D, Pantel K: HER2 status of circulating tumor cells in patients with metastatic breast cancer: a prospective, multicenter trial. Breast Cancer Res Treat 2010, 124:403-412.

19. Alix-Panabieres C, Pantel K: Circulating tumor cells: liquid biopsy of cancer. Clin Chem 2013, 59:110-118.

20. Pestrin M, Bessi S, Puglisi F, Minisini AM, Masci G, Battelli N, Ravaioli A, Gianni L, Di Marsico R, Tondini C, Gori S, Coombes CR, Stebbing J, Biganzoli L, Buyse M, Di Leo A: Final results of a multicenter phase II clinical trial evaluating the activity of single-agent lapatinib in patients with HER2-negative metastatic breast cancer and HER2-positive circulating tumor cells. A proof-of-concept study. Breast Cancer Res Treat 2012, 134:283-289.

21. Bidard FC, Fehm $T$, Ignatiadis $M$, Smerage JB, Alix-Panabières $C$, Janni $W$, Messina C, Paoletti C, Müller V, Hayes DF, Piccart M, Pierga JY: Clinical application of circulating tumor cells in breast cancer: overview of the current interventional trials. Cancer Metastasis Rev 2013, 32:179-188.

22. Riethdorf S, Fritsche H, Müller V, Rau T, Schindlbeck C, Rack B, Janni W, Coith C, Beck K, Jänicke F, Jackson S, Gornet T, Cristofanilli M, Pantel K: Detection of circulating tumor cells in peripheral blood of patients with metastatic breast cancer: a validation study of the Cell Search system. Clin Cancer Res 2007, 13:920-928.

23. Kraan J, Sleijfer $S$, Strijbos MH, Ignatiadis $M$, Peeters $D$, Pierga JY, Farace $F$, Riethdorf S, Fehm T, Zorzino L, Tibbe AG, Maestro M, Gisbert-Criado R, Denton G, de Bono JS, Dive C, Foekens JA, Gratama JW: External quality assurance of circulating tumor cell enumeration using the Cell Search ((R)) system: A feasibility study. Cytometry B Clin Cytom 2011, 80:112-118.

24. Tibbe AG, Miller MC, Terstappen LW: Statistical considerations for enumeration of circulating tumor cells. Cytometry A 2007, 71:154-162.

25. Pierga J, Bidard F, Andre F, Petit T, Dalenc F, Delozier T, Romieu G, Bonneterre J, Ferrero J, Kerbrat P, Martin A, Viens P: Early drop of circulating tumor cells (CTC) and increase of circulating endothelial cells (CEC) during neoadjuvant chemotherapy (CT) combined with bevacizumab in HER2-negative inflammatory breast cancer (IBC) in multicenter phase II trial BEVERLY 1. J Clin Oncol 2011, 29:10510.

26. Pierga JY, Petit T, Delozier T, Ferrero JM, Campone M, Gligorov J, Lerebours F, Roché $H$, Bachelot T, Charafe-Jauffret E, Pavlyuk M, Kraemer S, Bidard FC, Viens $P$ : Neoadjuvant bevacizumab, trastuzumab, and chemotherapy for primary inflammatory HER2-positive breast cancer (BEVERLY-2): an open-label, single-arm phase 2 study. Lancet Oncol 2012, 13:375-384.
27. Riethdorf S, Mueller V, Mauermann O, Rau T, Loibl S, Eidtmann H, Solbach C, Tesch H, Schrader I, Kittel K, Fehm T, Holms F, Untch M, von Minckwitz G, Pantel $\mathrm{K}$ : Changes in circulating tumor and endothelial cells in peripheral blood of patients treated in the Neoadjuvant Chemotherapy Plus Targeted Treatment Breast Cancer Study "GeparQuinto". Cancer Res 2010, 70:PD04-6.

28. Landis J, Koch G: A one-way component of variance model for categorical data. Biometrics 1977, 33:671-679.

doi: $10.1186 /$ bcr3647

Cite this article as: Ignatiadis et al.: International study on inter-reader variability for circulating tumor cells in breast cancer. Breast Cancer Research 2014 16:R43.

\section{Submit your next manuscript to BioMed Central and take full advantage of:}

- Convenient online submission

- Thorough peer review

- No space constraints or color figure charges

- Immediate publication on acceptance

- Inclusion in PubMed, CAS, Scopus and Google Scholar

- Research which is freely available for redistribution 